\title{
PERCEPCIONES SOBRE ACTITUDES Y DESEO SEXUAL EN PERSONAS QUE COHABITAN EN PAREJA CON MÁS DE DOS AÑOS DE RELACIÓN
}

\author{
Perceptions of attitudes and sexual desire in people cohabiting \\ as a couple with more than two Years of relationship
}

\section{Rosaura Gutiérrez Valerioa, Dunia Chávez Esponda ${ }^{b}$ y Deseado Guzmán Esquea}

Recibido: 24 de enero, 2020 • Aprobado: 30 de marzo, 2020

Cómo citar: Gutiérrez Valerio R, Chávez Esponda D, Guzmán Esquea D. Percepciones sobre actitudes y deseo sexual en personas que cohabitan en pareja con más de dos años de relación. cysa [Internet]. 17 de junio de 2020 [citado 17 de junio de 2020];4(2):21-36. Disponible en: https://revistas.intec.edu.do/index.php/cisa/article/view/1766

\section{Resumen}

La sexualidad humana constituye una parte sumamente importante en la vida de las personas, sin embargo, para muchas de ellas se convierte en un tema lleno de mitos y tabúes. Este trabajo tuvo como objetivo explorar la percepción que tenían las parejas con más de dos años de convivencia sobre el deseo y actitudes sexuales como punto de partida para visibilizar las creencias, las conductas aceptadas y rechazadas que pueden estar influenciando en el disfrute o en la disfuncionalidad de una acoplada relación conyugal. Se aplicaron cuestionarios a una muestra de 52 personas del Cantón Puyo, Ecuador. Se incluyeron variables como: edad, sexo, tiempo de relación con la pareja, edad que tenía cuando tuvo su primera relación, si era mujer: situación en su ciclo reproductivo (menstruación, amenorrea, menopaúsica, embarazo) y nivel de estudios. Se utilizaron gráficos de barras, tablas de contingencia y pruebas Chi cuadrado. Los resultados mostraron que, en el campo del deseo, las actitudes sexuales y la fantasía, los hombres manifestaron que les gusta el sexo con mayor frecuencia que las mujeres y que sienten mucho placer o disfrute al pensar en el sexo. En cambio, las mujeres declararon que raramente o algunas veces tienen pensamientos

a Instituto de formación docente Salomé Ureña, Recinto Urania Montás. República Dominicana. Correo-e: rosaura.valerio@isfodosu.edu.do ORCID: 0000-0001-9920-1723

\begin{abstract}
Human sexuality is an extremely important part of people's lives; however, for many of them it becomes a subject full of myths and taboos. This work aimed to explore the perception that couples with more than two years of living with sexual desire and attitudes had as a starting point to make visible the beliefs, accepted and rejected behaviors that may be influencing enjoyment or dysfunctionality of a coupled marital relationship. Questionnaires were applied to a sample of 52 people from the Puyo Canton. Variables were included such as: age, sex, time of relationship with the couple, how old she was when she had her first relationship, if she was a woman: situation in her reproductive cycle (menstruation, amenorrhea, menopause, pregnancy) and level of education. Bar charts, contingency tables and Chi square tests were used. The results showed that, in the field of desire, sexual attitudes and fantasy, men stated that they like sex more often than women and that they feel much pleasure or enjoyment when thinking about sex. On the other hand, women declared that they rarely or sometimes have sexual thoughts, observing a lesser motivation for sexual activity, whether due to work or financial problems in many of the cases surveyed.

\footnotetext{
b Universidad Estatal Amazónica. Ecuador

Correo-e: dchavez@uea.edu.ec, ORCID: 0000-0003-2736-6072

${ }^{\mathrm{c}}$ Hospital Metropolitano de Santiago. República Dominicana.
}

Correo-e: deseado@gmail.com
\end{abstract}


sexuales, observándose una menor motivación por la actividad sexual, ya sea por problemas laborales o financieros, en muchos de los casos encuestados. Estos resultados permitirán reforzar en los talleres realizados, los temas que generaron distanciamientos en las parejas.

Palabras clave: actitud; deseo; sexo; pareja; convivencia.

\section{Introducción}

La sexualidad humana es vista por muchos estudiosos de la materia como una parte sumamente importante en la vida de las personas. Cuando se mira la pirámide de Abraham Maslow, una de las necesidades básicas que todo ser humano tiende a satisfacer es la del sexo. Según Balac el sexo es una actividad biológica, una función vital y no una mera opción que se ejerce al vaivén de factores y condicionantes externos. ${ }^{1}$

Para muchas parejas que han pasado la etapa de enamoramiento, con el transcurso del tiempo, el deseo y la fantasía ocupan un segundo plano en la relación, haciendo rutinarios los momentos y llenando de monotonía espacios y contactos que fueron puntos de encuentros para el deleite y el disfrute, en la exploración de su propio yo a través de la sexualidad. De acuerdo a Parra et al., ${ }^{2}$ la rigidez dentro de los subsistemas (conyugal y parental), la falta de claridad, los mitos y tabúes en torno a las fantasías y el deseo, la influencia de las culturas y la religión muchas veces son variables que ponen en juego la libertad de expresión de una sexualidad libre de prejuicios.

La experiencia del deseo en el ser humano, indiscutiblemente, pasa por una combinación neurohormonales y cognitivo-emocionales que predispone al individuo para el disfrute y el placer. Leiblum y Rosen, citados en Zubeidat et al., ${ }^{3}$ señalaron que: "el deseo sexual sería resultado de la combinación de un adecuado funcionamiento neuro-endocrino con una estimulación sexual provocada por dos fuentes de activación, una individual (fantasías, ideaciones, vaso congestión genital, etc.) y otra
These results will reinforce in the workshops carried out, the issues that generated distancing in couples.

Keywords: Attitudes; wish; sex; couples; coexistence.

ambiental (como estar acompańado por una pareja deseada con luz ligera, por ejemplo), estando determinado por procesos sexuales intrapsíquicos e interpersonales". ${ }^{3}$

Por su parte Sierra et. al., ${ }^{4}$ citando a Kaplan, define el deseo como "las sensaciones que motivan a un individuo a iniciar o ser receptivo a una estimulación sexual"4. Estos autores hablan de deseo sexual provocado por estimulación interna (por ejemplo, factores biológicos o pensamientos) y deseo sexual provocado por estimulación externa (por ejemplo, la visión de una pareja atractiva $)^{4}$.

Gómez ${ }^{5}$ afirmó que el deseo sexual tiene un origen claramente biofisiológico con una estructura anatómico-neuro-endocrina bastante perfilada. Su función, desde el punto de vista etiológico, se centra en la supervivencia de la especie a través de la reproducción. Sin embargo, el ser humano trasciende esta dependencia elemental y, desde un punto de vista psicológico, el deseo sexual se convierte en una de las motivaciones más importantes de la existencia. Desde nuestro punto de vista, el sistema motivacional constituye el nivel básico del deseo sexual, siendo este el resultado de la elaboración psicológica de aquel ${ }^{5}$.

A pesar de los aportes de varios estudios que dejan evidencias de los elementos que definen el deseo sexual, para su evaluación hace falta la búsqueda de diferentes variables multicausales que sincronicen con lo biológico, lo psicológico y lo cultural. De acuerdo a López ${ }^{6}$, la sexualidad es una de las más privadas e íntimas conductas humanas; hablar de ello, hasta hace poco, era algo que generaba vergüenza 
y cierto pudor. De hecho, en muchas culturas y religiones se ve como algo que se maneja con cierta distancia, por lo que ejercer la sexualidad sin temores, vergüenzas, mitos y falacias amerita aún de mucho trabajo de concienciación. Indudablemente que estos factores influyen, en el permiso y la libertad de desear, imaginar y fantasear, sobre la posibilidad de disfrutar y ejercer la sexualidad sin tabúes. Aceptar el cuerpo como fuente de placer, aceptar la intimidad como algo necesario y entender asumir sin culpabilidad, bienestar, placer y felicidad es importante para el desarrollo de una sexualidad sana.

El miedo y la culpa son vistos como elementos que debilitan el acercamiento y desinhibición para el disfrute, sin embargo, influyen poderosamente en el caso de las mujeres, que al estar con una pareja cercana y amorosa en la que se confía y se puedan sentir libres y seguras de hablar acerca de sus sueños, fantasías y necesidades permite tener una mejor respuesta sexual ${ }^{6}$. En culturas mexicanas, principalmente las tradicionales, a pesar de avances en varios aspectos de inclusión en espacios públicos, el ejercicio de la sexualidad sigue siendo un tema recriminado y visto solo como aspecto para la reproducción humana. Este elemento tiende a reproducirse en varias culturas por todo el continente latinoamericano, en donde la sexualidad ha estado muy ligada a cuestiones moralistas, y más allá de aparearse para parir, el reconocimiento de las necesidades, la exploración del propio cuerpo no es visto como elemento primordial en el relacionamiento de pareja.

A pesar de ser visto como algo prohibido en muchos sectores, sí es conocido cada vez más cómo las parejas acuden en busca de ayuda para resolver problemas de disfunción sexual, en lo que se inscribe el deseo sexual inhibido y otras patologías que lastiman la vida de la pareja.

De acuerdo con la Organización Mundial de la Salud, citado en Cordeiro" "La Salud Sexual es un estado de bienestar físico, emocional, mental y social en relación con la sexualidad; no solo la ausencia de enfermedad, disfunción o alteración. Requiere, además, un acercamiento positivo y respetuoso a la sexualidad, así como la posibilidad de mantener experiencias sexuales placenteras y seguras, libres de coerción, discriminación y violencia”. En ese sentido, los objetivos de la terapia sexual se conceptualizan de las siguientes maneras: "Educar sexualmente en valores y armonía personal; resolver la disfunción evitando el desplazamiento a otras áreas de la conducta; mejorar la capacidad de goce de la sexualidad como energía de vida; ampliar el significado de la sexualidad, desamurando de lo estrictamente genital". 7

Feixas et al., ${ }^{8}$ afirmaron que las terapias sistémicas de pareja producen resultados beneficiosos en aproximadamente dos tercios de los casos, y sus efectos son superiores a la ausencia de tratamiento. Además, cuando se logra implicar a ambos esposos en terapia conjunta para resolver sus problemas matrimoniales, existe una mayor probabilidad de éxito que si solo acude uno de ellos ${ }^{8}$.

Por otro lado, Laso9 planteó que en terapia de pareja, la evocación de las emociones se facilita porque se cuenta con la figura que las desencadena, el cónyuge: basta con pedir a ambos que manifiesten sus quejas para despertar una letanía a menudo imparable de resentimiento, ira, desprecio, miedo y lástima. El terapeuta tiene que apuntar no al contenido del conflicto, sino a la estructura de la relación que lo genera; y esta estructura obedece a las emociones que la canalizan, derivadas a su vez de las necesidades insatisfechas que cada miembro de la interacción intenta satisfacer, o al menos acallar con su conducta?

Según Cordeiro ${ }^{7}$, en su exposición en II Conferencia Internacional de Comunicación en Salud, en La Universidad Carlos III de Madrid: "gran parte de los pacientes con problemas sexuales podrían 
resolverlos si tuvieran la posibilidad de comportarse abiertamente como seres sexuales, de tener deseo sexual y si recibieran la información adecuada para afrontarlos". Debido a la importancia que reviste la temática, este trabajo tuvo como objetivo explorar la percepción que tienen las parejas con más de 2 años de convivencia sobre el deseo y actitudes sexuales, como punto de partida para visibilizar las creencias, las conductas aceptadas y rechazadas que pueden estar influenciando en el disfrute o en la disfuncionalidad de una acoplada relación conyugal, y con ello se pretenden reforzar, en los talleres realizados, los temas que generan distanciamientos en las parejas?

\section{Metodología}

La investigación se realizó en el Cantón Puyo, provincia de Pastaza, Ecuador. Se llevó a cabo un muestreo no probabilístico, de tipo incidental. En este tipo de muestreo el investigador determina deliberadamente qué individuos formarán parte de la muestra, tratando de escoger a los casos considerados típicamente representativos de la población. Los criterios de elección se basaron en el conocimiento teórico sobre el tema objeto de estudio. La muestra quedó compuesta por 52 personas, de las cuales 22 son hombres denominados en los análisis como: sexo $1(42,3 \%)$ y 30 son mujeres $(57,7 \%)$, denominadas como: sexo 2 .

Para la recolección de datos se aplicó el cuestionario CSFQ-14 Changues in Sexual Functioning Questionnaire (Keller et al., 2006, citado en López ${ }^{6}, 2013$, p. 45). Para el presente estudio, se utilizaron las mismas dimensiones usadas por la referida autora en su tesis de grado. En su aplicación se trabajaron solo tres sub-escalas: deseo, excitación-orgasmo y placer. Además de este instrumento se incluyeron de manera separadas informaciones correspondientes a: edad, sexo, tiempo de relación con la pareja, edad que tenía cuando tuvo su primera relación, si era mujer: situación en su ciclo reproductivo (menstruación, amenorrea, menopaúsica, embarazo) y nivel de estudios.

La aplicación del cuestionario se realizó de la siguiente manera. Se escogió como escenario un taller denominado: "Despertando nuestros deseos", cuyo objetivo era re-significar pautas de comportamiento que ponían en juego el relacionamiento y enfriaba la relación en la intimidad. Fueron seleccionadas todas las parejas que participaban de talleres organizados sobre temas de parejas, fueron informadas de la utilidad de dicha investigación para futuras acciones individuales y procedieron a responder los cuestionarios. Por otro lado, se seleccionaron parejas que de alguna manera tenían cierto vínculo con la investigadora o unas colegas quienes acompańaban procesos de talleres de formación. Estas parejas o personas en parejas deberían cumplir el requisito de que su relación contara con más de dos años de convivencia juntos y que formaran parte del Cantón Puyo. Además, se incluyeron en la presente investigación los análisis que salieron en los grupos de trabajos.

Se diseńó la base de datos y se analizaron estadísticamente a través de gráficos de barras, pruebas de hipótesis chi cuadrado y tablas de contingencia. Para el procesamiento estadístico se utilizó el software SPSS, versión 18.0.

\section{Resultados}

La edad promedio de los participantes fue de aproximadamente 37 ańos, con una desviación estándar de 9 años. La edad promedio de su pareja fue aproximadamente 38 años y una desviación estándar de 9 años; 3 personas no respondieron a esta pregunta (tabla 1). 
Tabla 1. Media de la edad de la muestra

\begin{tabular}{|c|c|c|c|}
\hline \multicolumn{2}{|l|}{} & Edad & $\begin{array}{c}\text { Edad de } \\
\text { pareja }\end{array}$ \\
\hline N & Válido & 52 & 49 \\
\hline & Perdidos & 0 & 3 \\
\hline Media & & 37,15 & 37,86 \\
\hline Mediana & & 36,00 & 37,00 \\
\hline Desviación estándar & 9,017 & 9,885 \\
\hline
\end{tabular}

Fuente: SPSS Versión 22 (2014).

En cuanto al tiempo reportado en la relación manifestaron entre 2 y 33 años, siendo 4, 10 y 14 años donde se observa un mayor porcentaje de la muestra (figura 1).

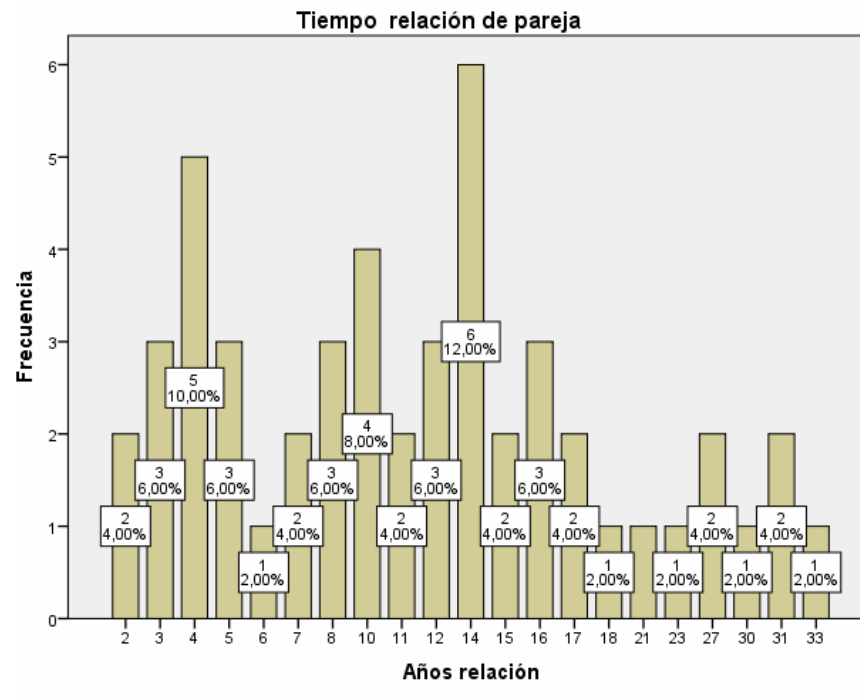

Figura 1. Tiempo de relación de las parejas

Fuente: SPSS Versión 22 (2014).
Otro dato interesante es el representado en la figura 2, donde se muestra el nivel de estudios de los participantes: 1 persona reportó estudios de primaria, para un 1,92 \%; 8 de los participantes seńalaron que aprobaron un nivel secundario, para un 15,38 \%; 12 de ellos respondieron que habían recibido solo alguna formación técnica, para un 23,08 \% mientras que la mayor cantidad de participantes (32 personas) reportaron haber concluido alguna formación universitaria, para un 59,62 \%.

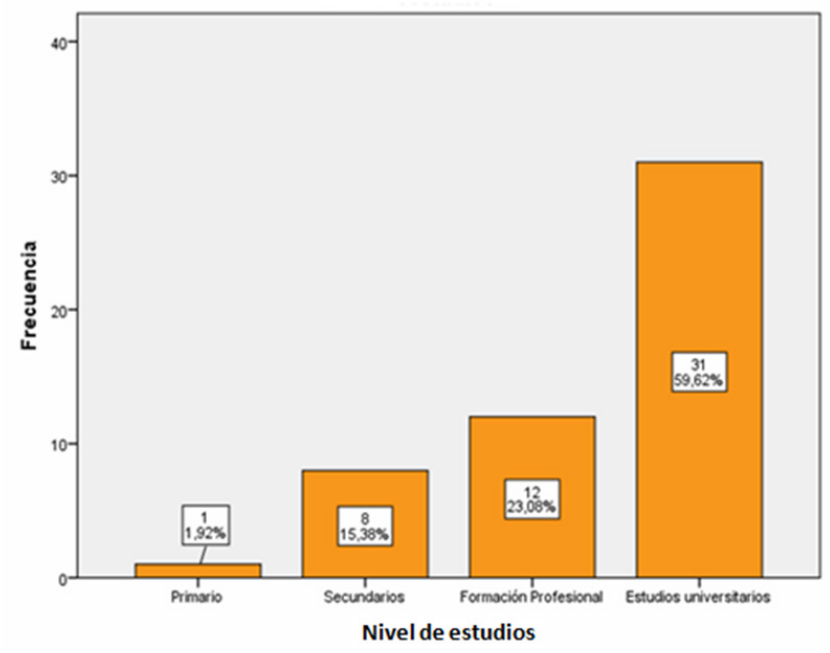

Figura 2. Nivel de estudio de los participantes.

Fuente: SPSS Versión 22 (2014).

Por otra parte, se analizó la edad de la primera experiencia sexual de los participantes. En tal sentido, se observó que la primera relación sexual fue notificada en el rango de 12 a 40 años, siendo 16 y 17 ańos las edades más frecuentes de su primera experiencia sexual, cuando todavía eran menores de edad. Esto puede influir de manera relevante en la estabilidad de la pareja y su deseo sexual. Seguidamente se observa que los valores 18,19 y 20 años fueron las edades que presentaron mayor número de personas y más elevados porcentajes (figura 3 ). 


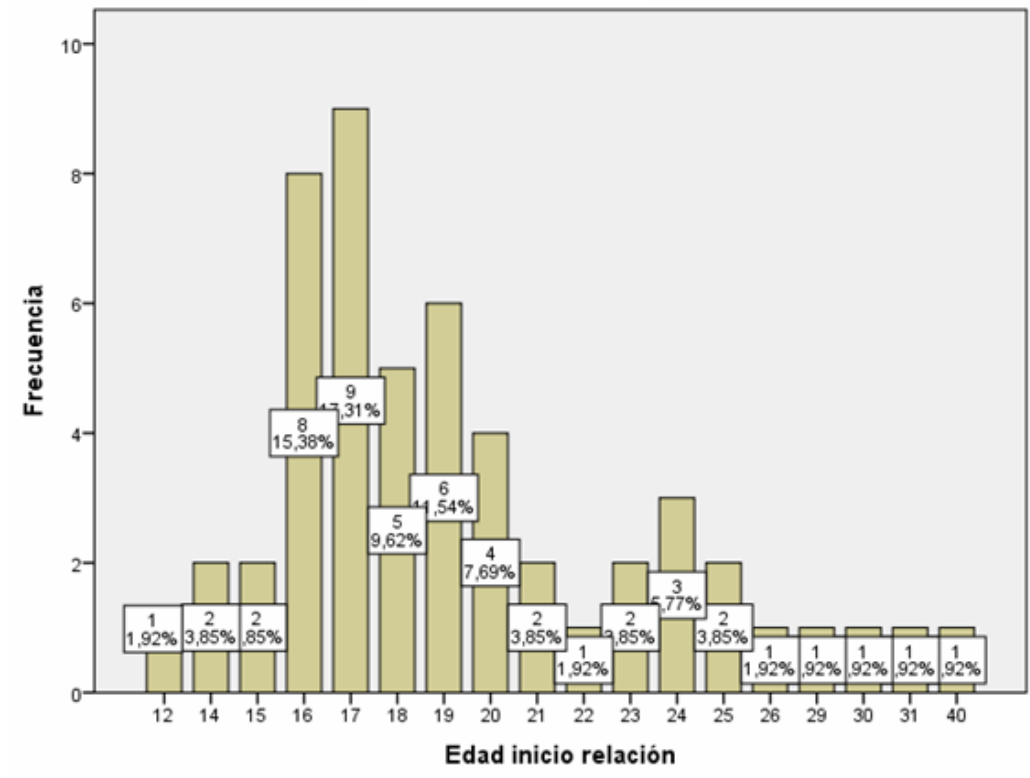

Figura 3. Edad de inicio de la relación

Fuente: SPSS Versión 22 (2014).

El ciclo en el que se encontraban las mujeres participantes es como sigue a continuación: 13 mujeres de las participantes, para un 43,33\%, manifestaron tener menstruación regularmente; 9, para un $30 \%$, señalaron que tienen menstruación irregularmente; 1 , en cambio, reportó estar en la menopausia, para el 3,33\%; 16 (67\%) de ellas señalaron no tener menstruación y solo 2 , para un $6,67 \%$, se declararon en estado de embarazo (figura 4).

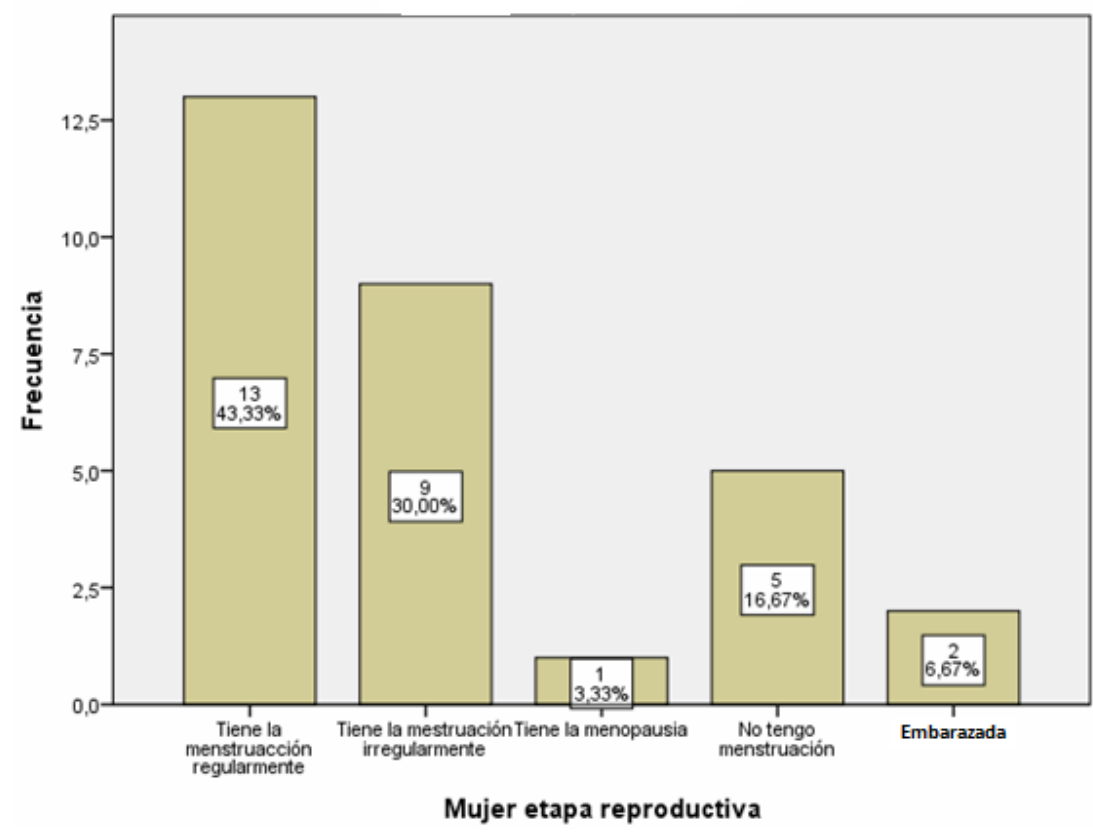

Figura 4. Etapa reproductiva de la mujer Fuente: SPSS Versión 22 (2014). 
Para analizar el comportamiento y actitudes sexuales entre hombres y mujeres, además del análisis de las frecuencias, se utilizó la prueba de hipótesis chi cuadrado con sus correspondientes tablas de contingencia o tablas cruzadas. A nivel descriptivo el mayor porcentaje fue $45,10 \%$, los cuales manifestaron tener a menudo algún tipo de actividad sexual, mientras que un $1,96 \%$ señaló que nunca (figura 5). Refiriéndose a la frecuencia en que le gustaría tener actividad sexual, el 57,69 \% manifestó que a menudo, un $11,54 \%$ raramente, $25 \%$ algunas veces y diario el 5,77 \% (figura 6). Se observa, al comparar entre el hombre y la mujer esta actitud hacia la frecuencia, que el 32,69 \% de los hombres manifestó a menudo, mientras que las mujeres solo un $25 \%$. En la frecuencia "raramente" el 7,69 \% de las mujeres estaba en esta frecuencia y solo un $3,85 \%$ de los hombres (figura 7). Los resultados de la prueba chi cuadrado mostraron diferencias significativas con un valor $\mathrm{p}=0.082$, para un $10 \%$ de significación y un $90 \%$ de confiabilidad (tabla 2).

¿Con qué frecuencia mantiene actividad sexual (relaciones sexuales, masturbación)?

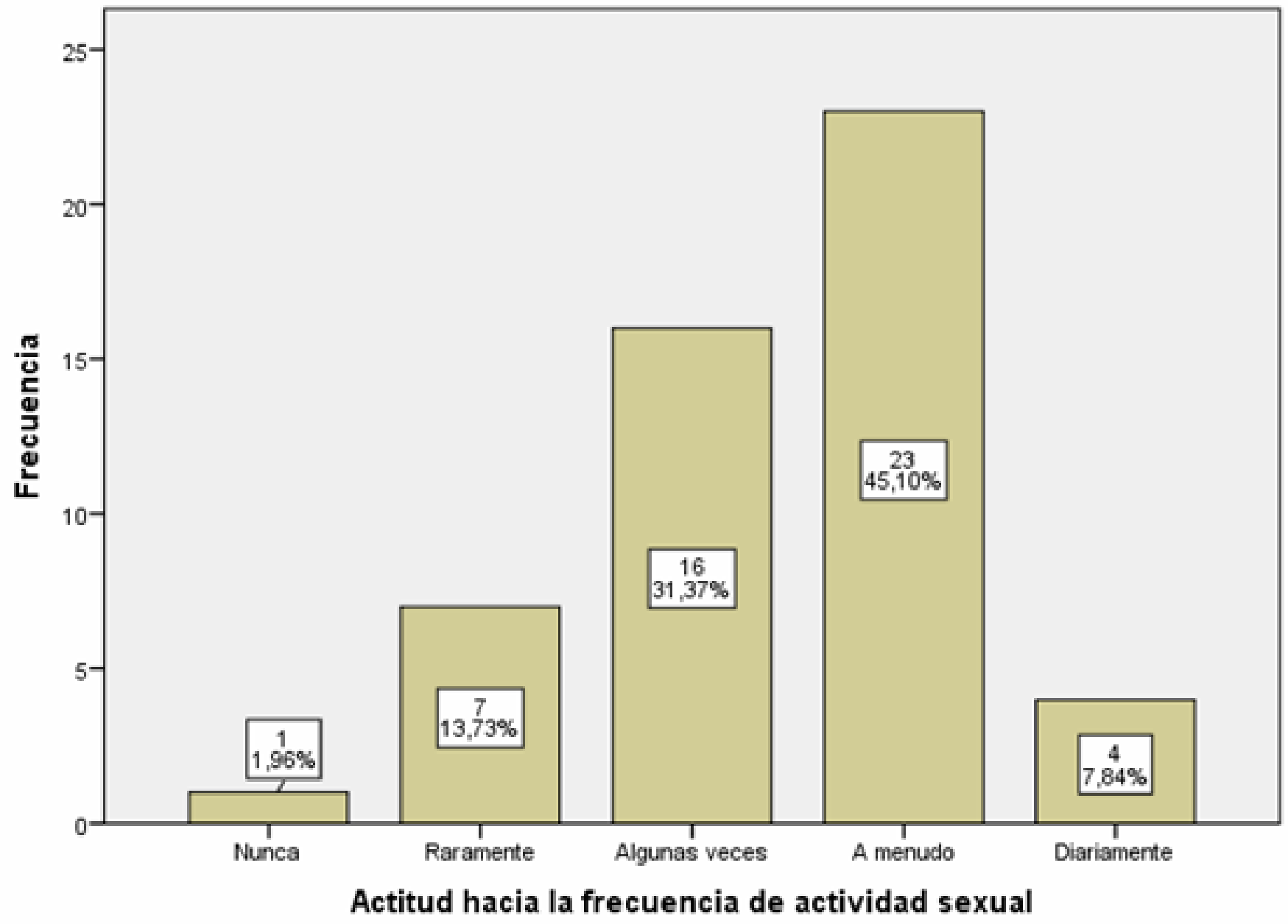

Figura 5. Frecuencia que mantienen actividades sexuales Fuente: SPSS Versión 22 (2014). 


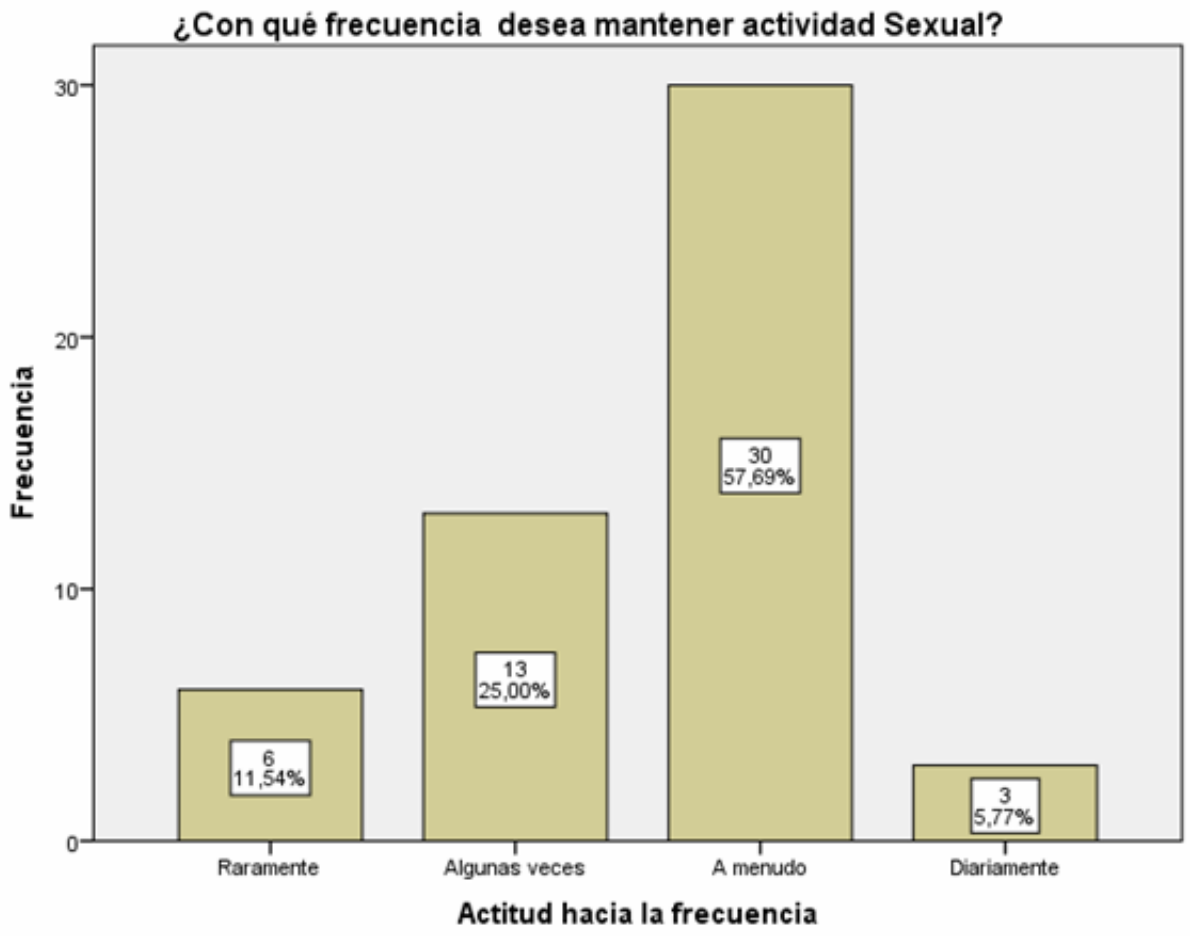

Figura 6. Deseos de la frecuencia de actividad sexual Fuente: SPSS Versión 22 (2014).

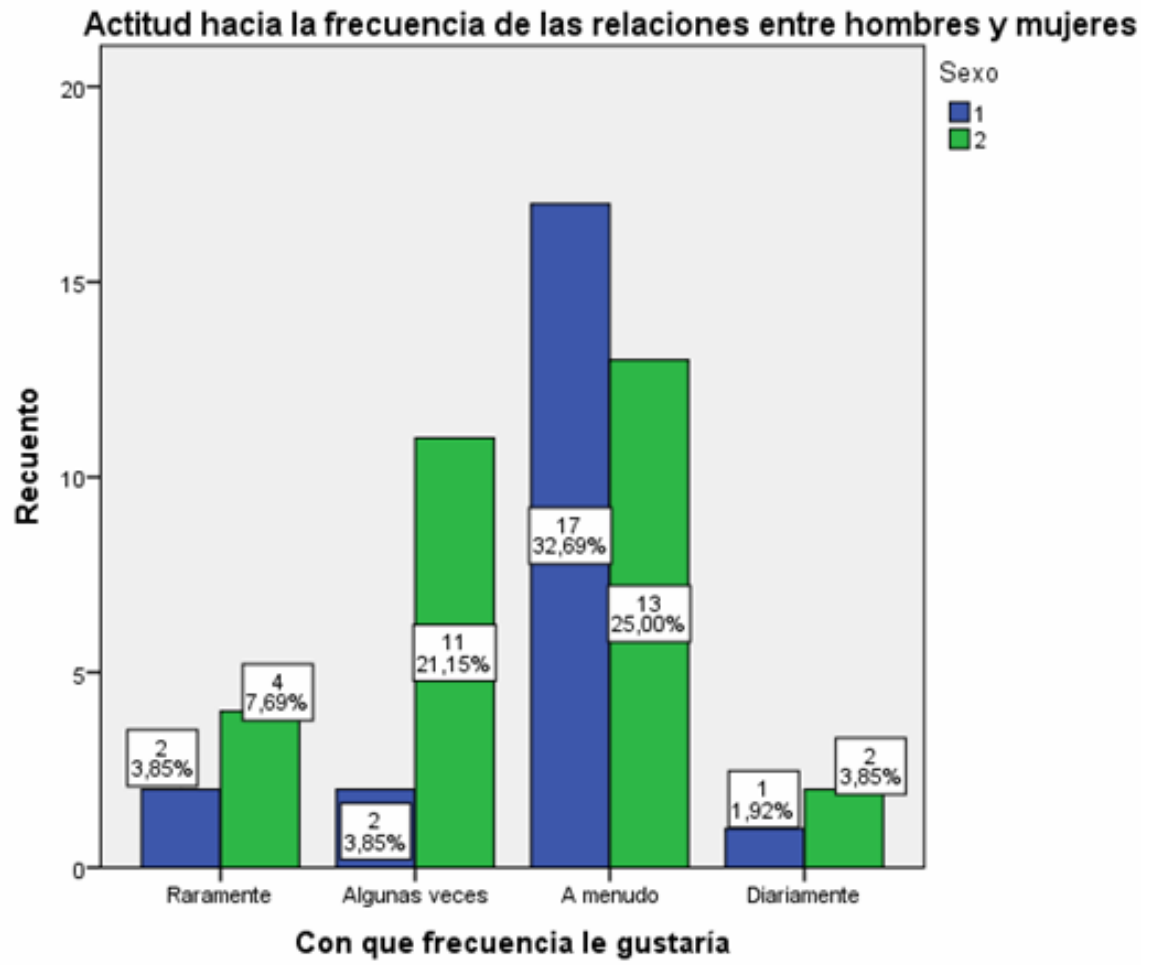

Figura 7. Comparación entre actitud hombre y mujer sobre frecuencia Fuente: SPSS Versión 22 (2014).

28 | Ciencia y Salud 2020; 4(2, mayo-agosto): 21-36 • Artículo original 
Tabla 2. Resultados de la prueba Chi-cuadrado

\begin{tabular}{|c|c|c|c|}
\hline & Valor & gl & $\begin{array}{c}\text { Sig. asintótica } \\
(2 \text { caras })\end{array}$ \\
\hline $\begin{array}{c}\text { Chi-cuadrado de } \\
\text { Pearson }\end{array}$ & $6,692 \mathrm{a}$ & 3 & $\mathbf{, 0 8 2}$ \\
$\begin{array}{c}\text { Razón de verosimilitud } \\
\text { Asociación lineal por } \\
\text { lineal }\end{array}$ & 7,178 & 3 &, 066 \\
N de casos válidos & 52 & 1 &, 119 \\
\hline
\end{tabular}

Fuente: SPSS Versión 22 (2014).

Analizando la frecuencia en la que se entretienen con pensamientos sexuales, el pensar hacer el amor y fantasías sexuales, 18 , para un $34,62 \%$, de los participantes manifestaron que "a menudo" tienen esta actitud; 5, para un 9,62\%, refirieron "nunca" y 4, para un 7,69 \%, de los entrevistados manifestaron a "diario" (figura 8). Analizando por sexo la apreciación de hombres y mujeres sobre este aspecto, de los 5 que reportaron nunca tener este tipo de actitud, 4 son mujeres y solo un hombre. De los 18 que seńalaron "a menudo" 11 son hombres y 7 mujeres $\mathrm{y}$ los que refirieron diariamente fueron 3 hombres $\mathrm{y}$ 1 mujer (figura 9). Estos resultados confirman los hallazgos encontrados en estudios anteriores donde la libertad del hombre está mejor condicionada para la expresión de este tipo de actitudes. Se encontraron diferencias significativas en la prueba chi cuadrado con un valor $\mathrm{p}=0.083$, para un $10 \%$ de significación y un $90 \%$ de confiabilidad (tabla3).

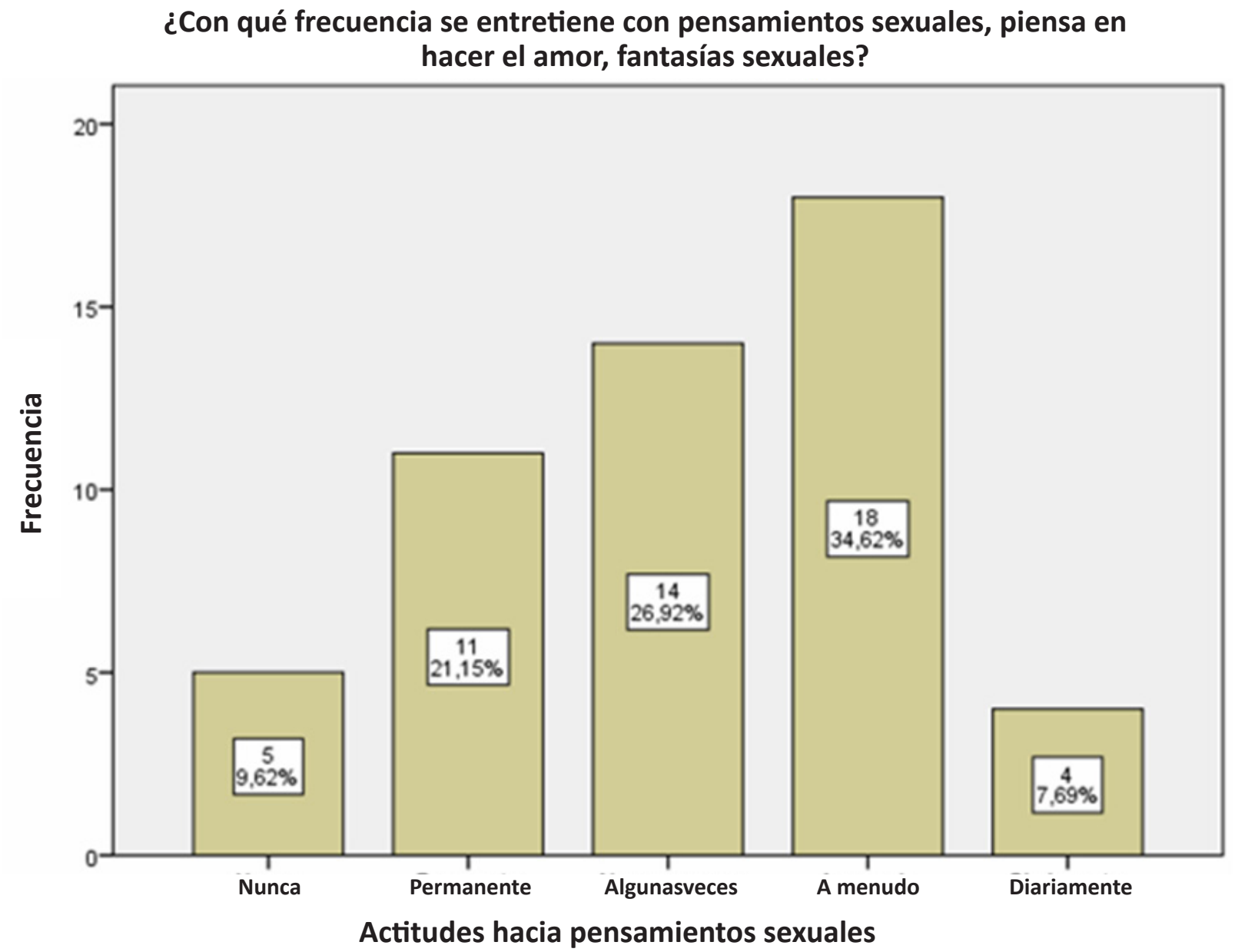

Figura 8. Frecuencia de pensamientos sexuales Fuente: SPSS Versión 22 (2014). 


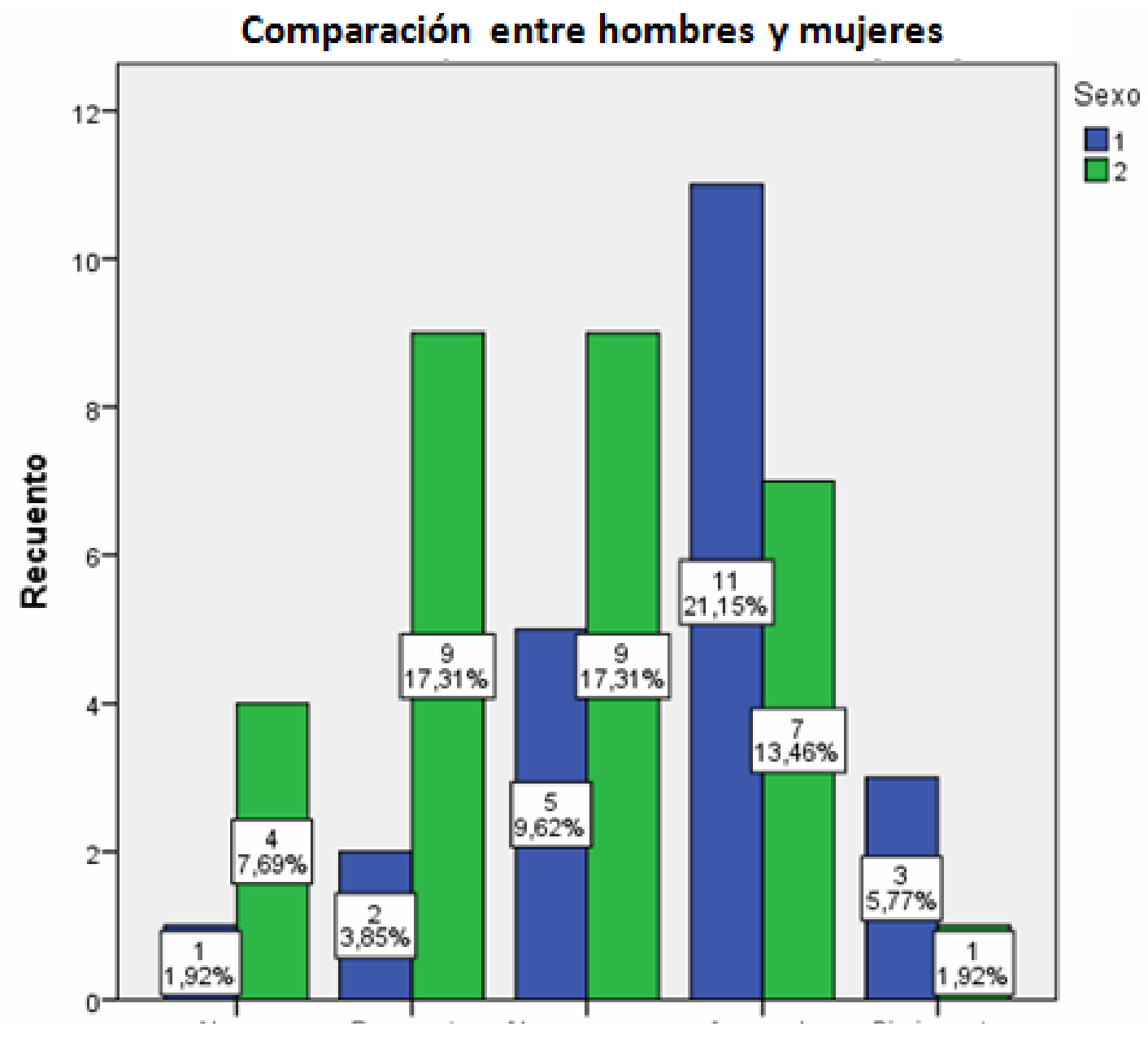

Figura 9. Comparación entre frecuencia de pensamientos de tipo sexual entre hombres y mujeres Fuente: SPSS Versión 22 (2014).

Tabla 3. Resultados de la prueba Chi-cuadrado

\begin{tabular}{|c|c|c|c|}
\hline & Valor & gl & $\begin{array}{c}\text { Sig. asintótica } \\
\text { (2 caras })\end{array}$ \\
\hline $\begin{array}{c}\text { Chi-cuadrado de } \\
\text { Pearson } \\
\text { Razón de } \\
\text { verosimilitud } \\
\text { Asociación lineal por } \\
\text { lineal }\end{array}$ & $8,251 \mathrm{a}$ & 4 & $\mathbf{0 8 3}$ \\
\begin{tabular}{c}
$\mathrm{N}$ de casos válidos \\
\hline
\end{tabular} & 52 & 1 &, 007 \\
\hline
\end{tabular}

Fuente: SPSS Versión 22 (2014).
Analizando el disfrute que tienen los participantes con la exposición a libros, películas, música o arte de contenido sexual; el 28,85 \% señaló que nunca se expone. Llama a la atención que varias personas no solo marcaron el ítem adecuado, sino que de igual manera escribieron frases como: no lo permito, o algo que denotaba aversión a la actitud de exponerse. En cambio, el 17,31\% y el 1,92\% manifestaron a menudo o a diario, respectivamente (figura 10). De los que marcaron "a menudo", 6 fueron hombres y 2 mujeres (figura 11). La opción "diariamente" fue seleccionada por un hombre. Los resultados de la prueba chi cuadrado mostraron diferencias significativas con un valor $\mathrm{p}=0.052$, para un $5 \%$ de significación y un $95 \%$ de confiabilidad (tabla 4). 


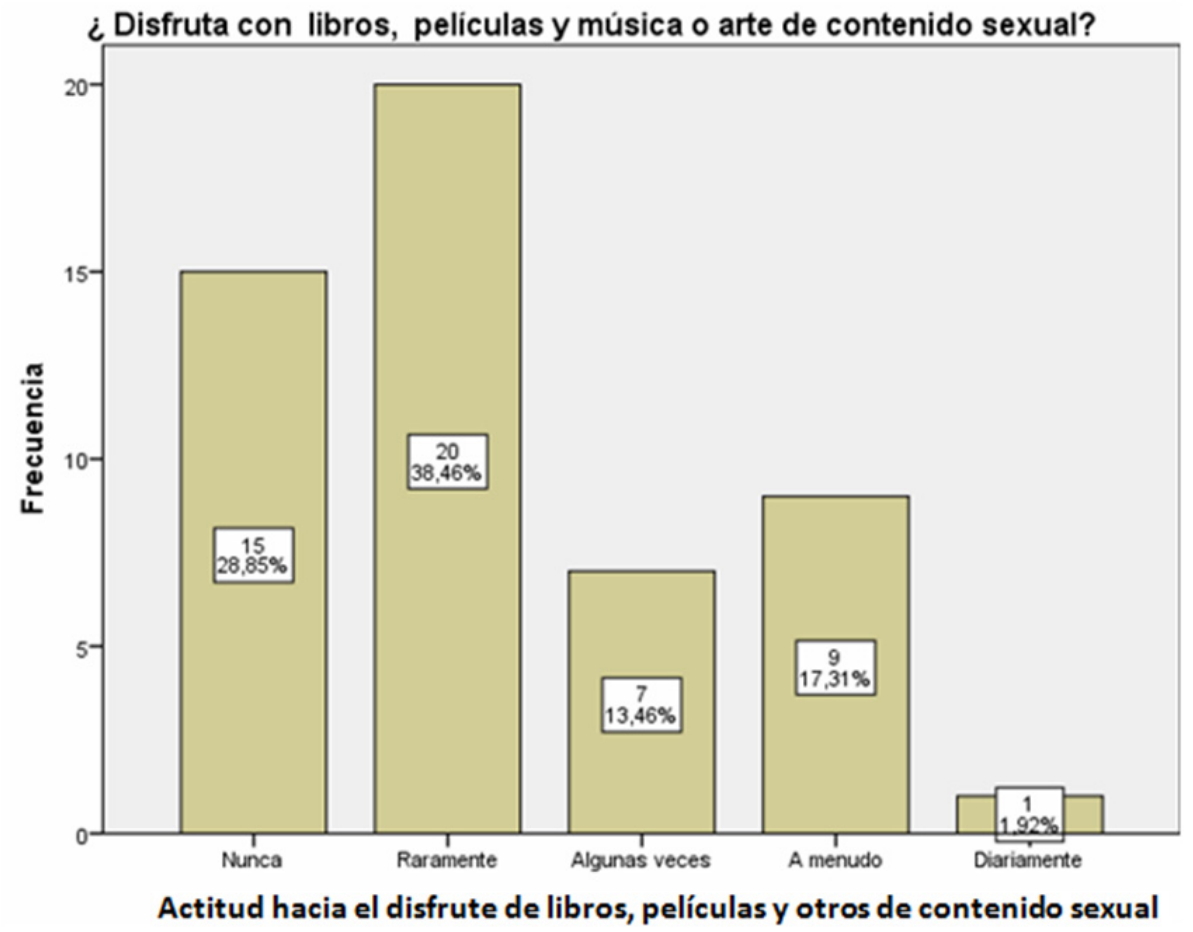

Figura 10. Disfrute de libros, películas y música contenido sexual Fuente: SPSS Versión 22 (2014).

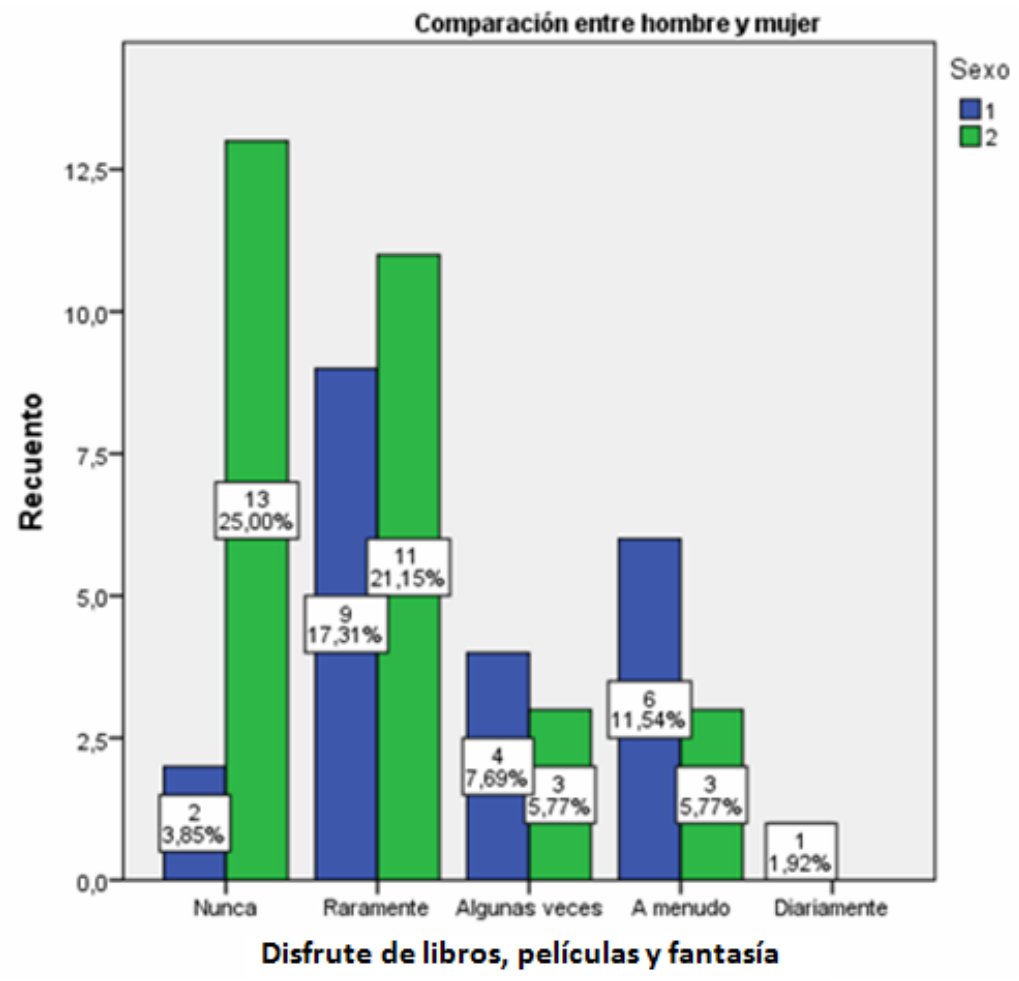

Figura 11. Comparación entre hombre y mujer sobre frecuencia de disfrute con libros y otros Fuente: SPSS Versión 22 (2014). 
Tabla 4. Resultados de la prueba chi cuadrado

\begin{tabular}{|c|c|c|c|}
\hline $\begin{array}{c}\text { Pruebas de } \\
\text { chi-cuadrado }\end{array}$ & Valor & gl & $\begin{array}{c}\text { Sig. } \\
\text { asintótica } \\
(2 \text { caras })\end{array}$ \\
\hline $\begin{array}{c}\text { Chi-cuadrado de } \\
\text { Pearson } \\
\text { Razón de } \\
\text { verosimilitud }\end{array}$ & $9,401 \mathrm{a}$ & 4 & $\mathbf{, 0 5 2}$ \\
$\begin{array}{c}\text { Asociación lineal } \\
\text { por lineal }\end{array}$ & 8,336 & 1 &, 004 \\
$\begin{array}{c}\text { N de casos } \\
\text { válidos }\end{array}$ & 52 & 4 &, 032 \\
\hline
\end{tabular}

Fuente: SPSS Versión 22 (2014).
El mucho disfrute o placer que genera algún tipo de fantasía acerca del sexo fue referido por el 26,92 $\%$ de las personas participantes, el 5,77 \% señaló muchísimo disfrute, en cambio el 19,23 y 17,31\% manifestó no tener ningún o poco placer y disfrute, según la figura 12. Al realizar la tabla cruzada de las personas que señalaron mucho placer o disfrute: el $17,31 \%$ se correspondía al sexo masculino y el $9,62 \%$ al sexo femenino. De igual manera de los que disfrutan muchísimo: $3,85 \%$ son hombres y $1,92 \%$ mujeres. En cambio, de los que no obtienen ningún disfrute o placer: $17,31 \%$ son mujeres y solo 1,92 \% son hombres (figura 13). De igual manera, el análisis de chi cuadrado mostró diferencias significativas, con un valor $\mathrm{p}=0.047$, para un $5 \%$ de significación y un $95 \%$ de confiabilidad (tabla 5).

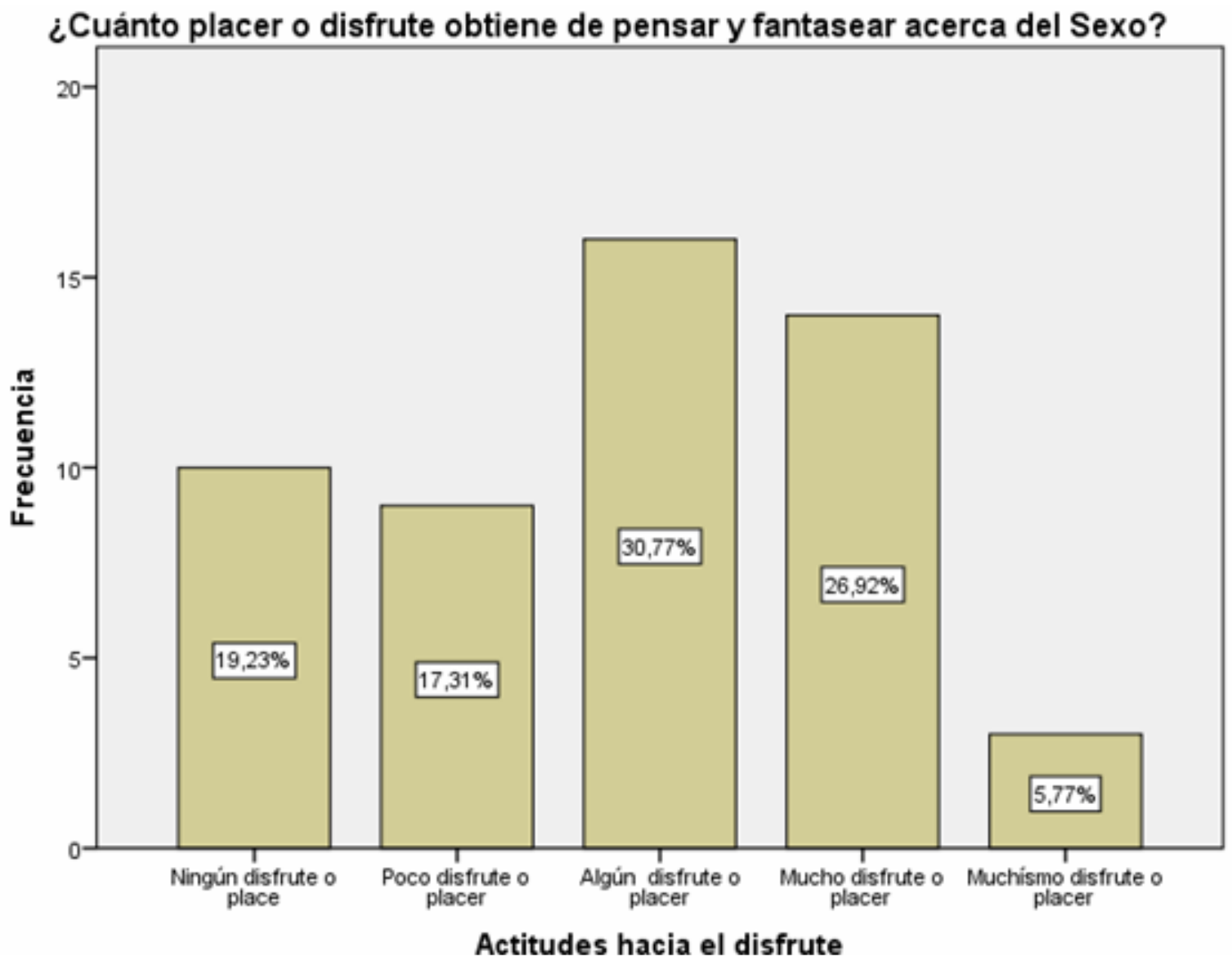

Figura 12. Disfrute que se obtiene de pensar y fantasear sobre sexo Fuente: SPSS Versión 22 (2014) 


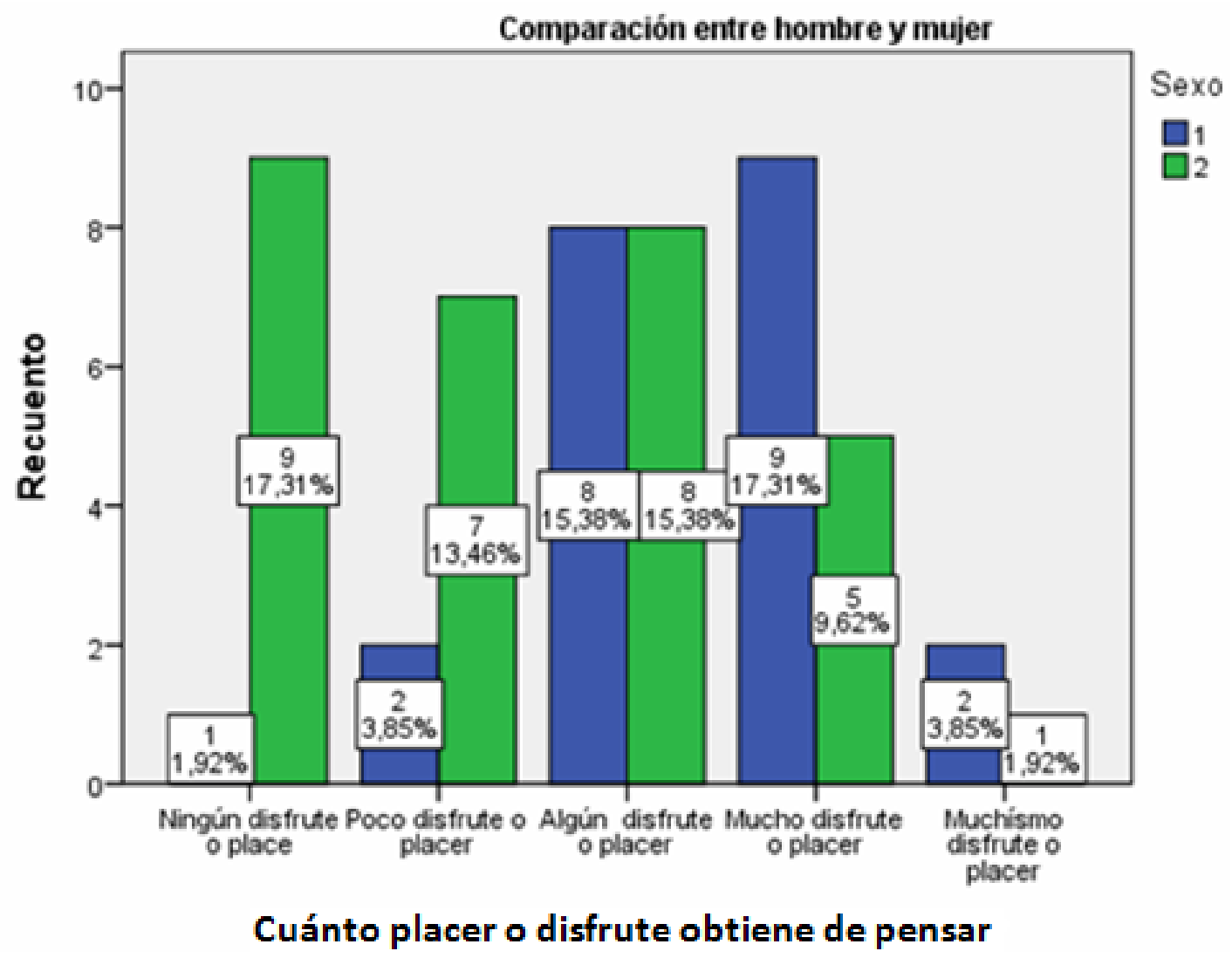

Figura 13. Comparación entre hombres y mujeres sobre el placer en pensar en el sexo Fuente: SPSS Versión 22 (2014).

Tabla 5. Resultados de la prueba chi cuadrado

\begin{tabular}{|c|c|c|c|}
\hline & Valor & $\mathrm{gl}$ & $\begin{array}{c}\text { Sig. } \\
\text { asintótica } \\
\text { (2 caras) }\end{array}$ \\
\hline $\begin{array}{c}\text { Chi-cuadrado } \\
\text { de Pearson }\end{array}$ & $9,652 \mathrm{a}$ & 4 & ,047 \\
\hline $\begin{array}{c}\text { Razón de } \\
\text { verosimilitud }\end{array}$ & 10,566 & 4 & ,032 \\
\hline $\begin{array}{c}\text { Asociación } \\
\text { lineal por } \\
\text { lineal }\end{array}$ & 8,975 & 1 & ,003 \\
\hline $\begin{array}{c}\mathrm{N} \text { de casos } \\
\text { válidos }\end{array}$ & 52 & & \\
\hline
\end{tabular}

Fuente: SPSS Versión 22 (2014).

\section{Discusión}

Los resultados obtenidos sobre la percepción que tienen las personas que conviven en parejas por más de dos años, coinciden con los planteados por López ${ }^{6}$ quien detectó en los hombres una mayor actitud positiva a la actividad sexual, fantasía y expresión del deseo.

Las diferencias que arrojan el disfrute a la exposición de la literatura de contenido sexual, erótico; el placer que obtienen al pensar en alguna actividad sexual; la frecuencia de tener pensamientos sexuales; la actitud hacia la frecuencia de tener relaciones, pueden estar relacionados a lo manifestado por Santos y Sierra $^{10}$, quienes afirman que las mujeres que discuten y toman en cuenta de sus necesidades sexuales son estigmatizadas como "zorras". Para las 
culturas que promueven y ven mal la exteriorización de las necesidades sexuales de las mujeres, les parecería mejor "guardar, cierto rescate para la expresión" y lo que no significa una pauta de tal cual expresa el estudio ${ }^{10}$.

Estas suposiciones colocadas por estos mismos autores, podrían lacerar la importancia que reviste el asertividad para la sexualidad; por estar relacionada al componente de respuesta sexual, deseo y satisfacción sexual. Esta pauta de comportamiento de "pasión dormida", no solo fue percibida en el taller realizado en parejas con más de 10 años de unión conyugal, sino que en parejas más jóvenes se obtuvo confesiones tales como: "hace tiempo nada nos calienta, ni tenemos casi intimidad sexual". ${ }^{10}$

Muchas situaciones conflictuadas fueron relacionadas con la crianza de los hijos. Las cuestiones laborales y económicas resaltaron como quejas que estorbaban el deseo de pensar en el otro "ya el sexo ni es alivio para mí, más bien es un asco y una carga", eran otras de las frases que de manera más íntima podían expresar algunas mujeres. Estos resultados concuerdan con los obtenidos por Saiz ${ }^{11}$ quien afirmó que la vida sexual de una pareja también puede verse condicionada por la inestabilidad financiera; la crisis económica no solo desestabiliza a los mercados de valores, sino también a las relaciones afectivas; cada vez más parejas se disuelven ante una mala situación financiera.

Los resultados obtenidos mostraron en evidencia lo importante y necesario que sería el abordaje de esta temática, donde se tome en consideración la complejidad que interviene en su desarrollo. Tal como se enunció al inicio de este trabajo, es común recibir en busca de ayuda personas que, en convivencia de pareja, la rutina y el desgano son parte del día a día, agregando ingredientes a pautas disfuncionales que se repiten de generación en generación, así como el miedo y la culpa que generan al hablar de estos temas impiden una mejor respuesta sexual (Cruz et al., $\left.{ }^{12}\right)$. De igual manera, es importante tomar en cuenta en el diagnóstico, un enfoque sistémico por la misma conformación de las relaciones en las parejas y sus historias de aprendizajes en el contexto de sus propios sistemas de origen, no solo abordar la problemática separada del todo que puede estar influyendo en el síntoma ${ }^{12}$.

Los resultados coinciden con los obtenidos por Yela ${ }^{13}$ quien mostró que en los hombres existe una gran discrepancia entre lo que se desea y se valora en la pareja a corto plazo frente al largo plazo, en las mujeres existe una gran similitud entre lo más valorado en ambos tipos de relaciones. ${ }^{13}$

Sostener una relación de pareja durante años, sin duda, implica un trabajo cotidiano. Trabajo que significa redefiniciones parciales, para dejar estables algunos perfiles de la relación. El pasaje de años hace variar los estilos relacionales amorosos, las formas de expresión afectiva, las necesidades, expectativas de respuesta, actividades, gustos y preferencias, entre otras cosas. No se trata de que la persona con quien se formó pareja sea otra persona $\left(\right.$ Ceberio $\left.{ }^{14}\right)$.

Finalmente es interesante en esta dinámica, tener presente la importancia dentro de las culturas para aceptar el cuerpo como fuente de placer, aceptar la intimidad como algo necesario y entender y asumir sin culpabilidad, bienestar, placer y felicidad lo cual repercute en el desarrollo de una sexualidad sana. (Ceberio y Serebrinsky) ${ }^{15}$

\section{Conclusiones}

Se observó una alta disponibilidad de las personas en participar en el presente estudio y exponer sus actitudes ante un tema tan lleno de mitos y tabúes. Esto evidencia la apertura al crecimiento para reaprender nuevas pautas que promuevan el bienestar de las parejas. El nombre dado al taller "Despertando nuestros deseos", provocó cierta curiosidad en los 
participantes y, al evaluar algunos de los participantes, específicamente, hombres, manifestaron que era algo que estaban necesitando para salvar su vida de matrimonio, lo que hace suponer que actividades grupales podrían generar nuevas apreciaciones y aprendizajes que fomenten la asertividad sexual, indudablemente generando un clima de empatía y confianza que no desvalida a las personas, pero que contrastan las creencias que impiden el disfrute y el placer.

En las parejas que mantenían más de dos años de convivencia se observó que, en el campo del deseo, actitudes sexuales y fantasía, los hombres manifestaron que les gusta el sexo con mayor frecuencia que las mujeres y que sienten mucho placer o disfrute al pensar en el sexo. En cambio, las mujeres declararon que raramente o algunas veces tienen pensamientos sexuales, observándose una menor motivación por la actividad sexual, ya sea por problemas laborales o financieros, en muchos de los casos encuestados.

La sociedad a nivel de redes y comunicación ha avanzado considerablemente, sin embargo, el relacionamiento humano sigue su ritmo de des-confort por lo que hace falta la intervención de educadores y terapeutas sistémicos.

\section{Bibliografía}

1. BalacE. (2017). El sexo como necesidad natural. [Online]. GDA/El Tiempo Disponible en: http:/www.indicepr.com/noticias/2017/03/27/ style/69308/el-sexo-como-necesidad-natural/. [Accessed 27 Marz 2017]

2. Parra A, Galarza L, Plua, N. Sexualidad responsable vs tabúes sociales. Revista San Gregorio, 2018;21:75-87.

3. Zubeidat I, Ortega V, Del Villar C, Sierra J. Un estudio sobre la implicación de actitudes y fantasías sexuales en el deseo sexual de los adolescentes. Anales de Psicología, 2015;21(2):268-75

4. Sierra J, Zubeidat I, Carretero H, y Reina S. Estudio psicométrico preliminar del Test del Deseo Sexual Inhibido en una muestra española no clínica. International Journal of Clinical and Health Psychology, 2003;3(3):489-504.

5. Gómez J. El deseo sexual y sus trastornos: aproximación conceptual y etiológica. Anuario de sexología, 1995;1:45-66.

6. López E. (2013). Deseos y actitudes sexuales en parejas universitarias. (Tesis Master Oficial en Sexología, Universidad de Almería, España). Recuperado de http://repositorio.ual.es/bitstream/handle/10835/2356/Trabajo.pdf?sequence $=1 \&$ is Allowed $=y$

7. Cordeiro M. Comunicación sobre Sexualidad: Modelo PLISSIT. En: II Conferencia Internacional de Comunicación en Salud. Universidad Carlos III de Madrid; 2015. Disponible en: http://docplayer.es/41278059-Ii-conferencia-internacional-de-comunicacion-en-salud. html

8. Feixas G, Muñoz D, Compañ V, Montesano A. (2016). El modelo sistémico en la intervención familiar [Online]. Universitat de Barcelona. Departament de Personalitat, Avaluació ITractament Psicológics. Disponible en: http://diposit. ub.edu/dspace/bitstream/2445/31584/6/ Modelo_Sistemico_Enero2016.pdf. [Accessed 20 enero 2016]

9. Laso E. El amor en clave emocional: enamoramiento, desconfirmación y terapia de pareja. Redes: revista de psicoterapia relacional e intervenciones sociales. 2018;37:11-35.

10. Santos P, Sierra J. El papel del asertividad sexual en la sexualidad humana: una revisión sistemática”. International Journal of Clinical and HealthPsycchology. 2010;10(3):553-77. 
11. Saiz Y. ¿Cómo afecta la crisis a la pareja? [Online] 2012. Disponibleen:https://www.lavanguardia. com/estilos-de-vida/20120921/54350838524/ como-afecta-la-crisis-a-la-pareja.html

12. Cruz C, Romero A, Erari F de M. Indicadores de Deseo, Autoerotismo e Impulsividad Sexual en Mujeres de la Ciudad de México. Acta de Investigación Psicológica, 2013;3(1):1031-40.

13. Yela C. Análisis de la teoría de las Estrategias Sexuales en la población española. Psicothema, 2012;24(1):48-54.
14. Ceberio M. Hacia el trastorno de alienación conyugal o la simbiosis descalificatoria. Redes: revista de psicoterapia relacional e intervenciones sociales. 2011;31:87-110.

15. Ceberio MR, Serebrinsky H. Dentro y Fuera de la Caja Negra: Desarrollos del modelo sistémico en psicoterapia. (1 era edición). Buenos Aires: Psicolibro Ediciones; 2011. 